Supplement of Nat. Hazards Earth Syst. Sci. Discuss., 2, 7507-7519, 2014

http://www.nat-hazards-earth-syst-sci-discuss.net/2/7507/2014/

doi:10.5194/nhessd-2-7507-2014-supplement

(C) Author(s) 2014. CC Attribution 3.0 License.

(c) (i)

Natural Hazards and Earth System Sciences

Discussions

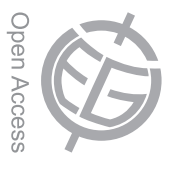

Supplement of

\title{
Earthquakes and depleted gas reservoirs: which comes first?
}

M. Mucciarelli et al.

Correspondence to: M. Mucciarelli (marco.mucciarelli@unibas.it) 


\section{Supplementary material}

\begin{tabular}{|c|c|c|c|c|c|c|c|c|c|}
\hline Well name & $\begin{array}{c}\text { Well } \\
\text { category }\end{array}$ & $\begin{array}{l}\text { Falls inside } \\
\text { CSS }\end{array}$ & $\begin{array}{l}\text { Falls inside } \\
\text { ISS }\end{array}$ & Sterile CSS & $\begin{array}{l}\text { Productive } \\
\text { CSS }\end{array}$ & $\begin{array}{c}\text { Sterile } \\
\text { background }\end{array}$ & $\begin{array}{l}\text { Productive } \\
\text { background }\end{array}$ & $\begin{array}{l}\text { Sterile } \\
\text { ISS }\end{array}$ & $\begin{array}{l}\text { Productive } \\
\text { ISS }\end{array}$ \\
\hline Albareto 1,2 & Sterile & ----- & ----- & & & 2 & & & \\
\hline Alfino 1 & Sterile & ------- & ------ & & & 1 & & & \\
\hline Alfonsine $1-18,20,21,22,26-29,33$ & Productive & ------ & ------ & & & & 26 & & \\
\hline Alfonsine $19,23,24,25,31,32,30$ & Sterile & ------- & ------- & & & 7 & & & \\
\hline Argenta 1 & Sterile & ------ & ----- & & & 1 & & & \\
\hline Baccarini 1 & Sterile & ITCS012 & ------ & 1 & & & & & \\
\hline Bagnolo in Piano 1, 2 & Sterile & ITCS049 & ------ & 2 & & & & & \\
\hline Bagnolo in Piano 3 & Ambiguous $^{\circ}$ & ITCS049 & ------ & & & & & & \\
\hline Bando 1, 2, 3 & Ambiguous $^{\circ 0}$ & ITCS012 & ITIS141 & & & & & & \\
\hline Bando 4, 6, 7 & Sterile & ITCS012 & ITIS141 & & & & & 3 & \\
\hline Baricella 1, 3 & Sterile & ITCS012 & ------ & 2 & & & & & \\
\hline Baricella 2 & Sterile & ------ & ------- & & & 1 & & & \\
\hline Baura 1 & Sterile & ITCS050 & ------- & 1 & & & & & \\
\hline Bevilacqua 1 & Sterile & ITCS051 & ---ב-ב-ב-ב- & 1 & & & & & \\
\hline Bignardi 1 & Sterile & ITCS051 & ITIS107 & & & & & 1 & \\
\hline Boreca 1 & Sterile & ------ & ------- & & & 1 & & & \\
\hline Boschi 24, 26, 27 & Sterile & ITCS012 & ------ & 3 & & & & & \\
\hline Boschi 25 & Ambiguous $^{\circ \circ}$ & ITCS012 & ------- & & & & & & \\
\hline Bottoni 1 & Ambiguous $^{\circ}$ & ITCS050 & ------ & & & & & & \\
\hline Bova 1, 3 & Ambiguous $^{\circ}$ & ITCS012 & ---ב-ב-ב- & & & & & & \\
\hline Bova 2, 4, 5 & Sterile & ITCS012 & ------- & 3 & & & & & \\
\hline Budrio 1 & Sterile & ------- & ------- & & & 1 & & & \\
\hline Campolungo 4 & Ambiguous $^{\circ}$ & ------ & ------ & & & & & & \\
\hline Camposanto 1 & Sterile & ------- & ------- & & & 1 & & & \\
\hline Camposanto 1 & Sterile & ITCS051 & ------- & 1 & & & & & \\
\hline Camurana 1, 2 & Sterile & ITCS051 & ITIS107 & & & & & 2 & \\
\hline Candiano 1 & Sterile & ITCS012 & ------- & 1 & & & & & \\
\hline Carpi 1 & Sterile & ----- & ------ & & & 1 & & & \\
\hline Casaglia 1 & Sterile & ITCS050 & ------ & 1 & & & & & \\
\hline Cascina Buzzoni 1 & Sterile & ITCS050 & ------- & 1 & & & & & \\
\hline
\end{tabular}




\section{Well name}

Cascina Nuova 1

Cascina S. Francesco 1

Case Borra 1, 2, 3

Case Chigi 1

Case Pinelli 1

Castelfranco Emilia 1,4

Cavone 1-5, 9-13, 15-17, 19, 21

Cavone 6, 14, 18, 20

Codigoro 1

Cona 1

Concordia 1

Consandolo 1

Consandolo C151

Corpo Reno $1^{*}$

Correggio 1-5, 7-25, 28, 29, 31, 34*, 40

Correggio 6, 26, 27, 30, 32, 33

Crevalcore 1

Diamantina 1, 8

Dogaro 1, 2

Dosso degli Angeli 1

Dosso degli Angeli 3*, 11*, 27*, 15*

Fabbrico 1

Ferrara 1

Francolino 1

Gaibana 1-4

Gallare 2

Gallare 3, 4, 5

Ghiarone 2

Lemizzone 1, 2

Longastrino 1, 3, 4, 5, 6

Longastrino 2

Madriole 1

Maiero 1, 2

Malalbergo 1, 29, 30, 33, 35, 36, 39

Malalbergo 23, 28, 32, 34, 37, 38, 40

\begin{tabular}{|c|c|}
\hline $\begin{array}{c}\text { Well } \\
\text { category }\end{array}$ & $\begin{array}{l}\text { Falls inside } \\
\text { CSS }\end{array}$ \\
\hline Sterile & ITCS050 \\
\hline Sterile & ------ \\
\hline Sterile & ------ \\
\hline Sterile & ITCS012 \\
\hline Sterile & -------- \\
\hline Sterile & -------- \\
\hline Productive & ITCS051 \\
\hline Sterile & ITCS051 \\
\hline Sterile & -------- \\
\hline Sterile & ITCS050 \\
\hline Sterile & ITCS051 \\
\hline Sterile & ITCS012 \\
\hline Ambiguous $^{\circ}$ & ITCS012 \\
\hline Ambiguous $^{\circ}$ & ITCS051 \\
\hline Productive & ITCS049 \\
\hline Sterile & ITCS049 \\
\hline Sterile & -------- \\
\hline Sterile & ITCS050 \\
\hline Sterile & ITCS051 \\
\hline Ambiguous $^{\circ}$ & -------- \\
\hline Productive & -------- \\
\hline Sterile & ITCS049 \\
\hline Sterile & ITCS050 \\
\hline Sterile & ITCS050 \\
\hline Ambiguous $^{\circ}$ & ITCS012 \\
\hline Sterile & ---- \\
\hline Productive & -------- \\
\hline Productive & ITCS050 \\
\hline Sterile & ITCS049 \\
\hline Sterile & ITCS012 \\
\hline Ambiguous $^{\circ}$ & ITCS012 \\
\hline Sterile & -ב---- \\
\hline Sterile & ITCS012 \\
\hline Ambiguous $^{\circ}$ & ITCS012 \\
\hline
\end{tabular}

Sterile CSS

Productive

css

Sterile

Productive

background

background

Sterile

1

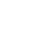

1

1

1

15

4

1

29

6 


\section{Well name}

Manara $3^{*}$

Mantello 1, 2, 3, 4

Medelana 1

Medolla 1

Mezzano 1

Migliarino 1

Migliarino 2

Minerbio 1-11, 13-19, 21-23, 37*, 52*

80*

Mirandola 1

Mirandola di Quarantoli 2

Molinella 1

Monestirolo 2

Monestirolo 3, 4, 5

Montalbano 1, 2, 4, 6, 9, 18

Montalbano 20

Montalbano 3, 5, 7, 8, 11, 20, 21

Muzza $1^{*}$

Muzza 2

Negrini 1

Nonantola 1

Novellara 1

Novi Modenese 1, 2, 3, 4

Ostellato 1

Palata Pepoli 1

Palazzo Majanti 1

Pavonara 1

Pieve di Cento 1

Pilastri 1, 2

Piombone 1

Poggetto 1

Poggio Rusco 1-5

Pomposa 3

Porotto 1

\begin{tabular}{cccr}
$\begin{array}{c}\text { Well } \\
\text { category }\end{array}$ & $\begin{array}{c}\text { Falls inside } \\
\text { CSS }\end{array}$ & $\begin{array}{c}\text { Falls inside } \\
\text { ISS }\end{array}$ & Sterile \\
Productive & ------ & ------- & \\
Sterile & ITCS012 & ------ & 4 \\
Sterile & ITCS050 & ------ & 1 \\
Sterile & ITCS051 & ITIS107 & \\
Sterile & ------ & ------ & \\
Ambiguous & & \\
Sterile & ITCS050 & ------ & \\
\hline ITCS050 & ------- & 1
\end{tabular}

Productive

css

\section{Sterile}

Productive Sterile Productive

4
1

1

Productive

Sterile

Sterile

Sterile

ITCS103

ITIS107

ITCS103

ITCS012

Productive ITCS012

Sterile ITCS012

Ambiguous $^{\circ 0}$ ITCS012

Sterile ITCS051

Sterile ITCS012

Productive

Sterile

Sterile

Sterile

Sterile

Sterile

Sterile

Sterile

Sterile

Sterile

Sterile

Sterile

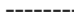

ITIS107

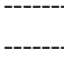

-------

$-----$

-------

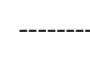

ITCS049

ITCS049

ITCS051

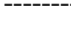

-------

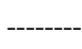

ITCS050

ITCS050

ITCS051

ITCS050

ITCS012

Ambiguous $^{\circ} \quad$ ITCS05

Sterile ITCS050

Productive

Sterile

ITCS050
24
1

1

1

1

- 1

- 2

5 


\section{Well name}

Porto Corsini 1

Porto Corsini 2, 3, 4, 10, 11, 12

Porto Corsini 5-8

Porto Verrara 1

Porto Verrara 2, 3

Ravarino 2

Ravenna 2, 12, 24, 32, 36, 37

Ravenna 3, 4, 7-11, 13-17, 20-23, 25,

28-31, 33-35, 38-52

Ravenna 37

Ravizza 1

Riccardina 1

Rio Saliceto 1

Rivara 1, 2

S. Alberto 1, 2

S. Biagio 1

S. Ermelinda 1

S. Felice sul Panaro 1

S. Giacomo 1

S. Giorgio di Piano 1

S. Giovanni 1

S. Maddalena 1

S. Martino in Spino 1

S. Pietro in Casale 1, 3-6, 8-11, 16, 17

18,20

S. Pietro in Casale 2, 7, 12-15, 19, 21 22

S. Possidonio 1

Sabbioncello 1-4, 6, 7, 8, 10

Sabbioncello $5,14,35^{*}, 23^{*}, 31^{*}, 13$,

Schiorsi 1

Schiorsi 1

Selva 2, 5-11, 17-21

Selva $3,4,14,15,16,23,24$

Sirocca 1

Spada 1

\begin{tabular}{|c|c|c|c|c|c|c|c|c|}
\hline $\begin{array}{c}\text { Well } \\
\text { category }\end{array}$ & $\begin{array}{l}\text { Falls inside } \\
\text { CSS }\end{array}$ & $\begin{array}{l}\text { Falls inside } \\
\text { ISS }\end{array}$ & Sterile CSS & $\begin{array}{l}\text { Productive } \\
\text { CSS }\end{array}$ & $\begin{array}{c}\text { Sterile } \\
\text { background }\end{array}$ & $\begin{array}{l}\text { Productive } \\
\text { background }\end{array}$ & $\begin{array}{l}\text { Sterile } \\
\text { ISS }\end{array}$ & $\begin{array}{l}\text { Productive } \\
\text { ISS }\end{array}$ \\
\hline Ambiguous $^{\circ}$ & ITCS012 & -------- & & & & & & \\
\hline Sterile & ITCS012 & -------- & 6 & & & & & \\
\hline Productive & ITCS012 & -------- & & 4 & & & & \\
\hline Ambiguous $^{\circ}$ & ITCS012 & ITIS141 & & & & & & \\
\hline Sterile & ITCS012 & ITIS141 & & & & & 2 & \\
\hline Productive & -------- & -------- & & & & 1 & & \\
\hline Sterile & ITCS012 & -------- & 6 & & & & & \\
\hline Productive & ITCS012 & -------- & & 39 & & & & \\
\hline Sterile & -------- & -------- & & & 1 & & & \\
\hline Productive & ITCS049 & -------- & & 1 & & & & \\
\hline Sterile & -------- & ------- & & & 1 & & & \\
\hline Sterile & ITCS049 & -------- & 1 & & & & & \\
\hline Sterile & ITCS051 & -------- & 2 & & & & & \\
\hline Sterile & ITCS012 & ------- & 2 & & & & & \\
\hline Sterile & ITCS051 & ITIS107 & & & & & 1 & \\
\hline Sterile & ITCS012 & ITIS141 & & & & & 1 & \\
\hline Sterile & ITCS051 & -------- & 1 & & & & & \\
\hline Productive & ITCS051 & ITIS107 & & & & & & 1 \\
\hline Sterile & -------- & -------- & & & 1 & & & \\
\hline Productive & ITCS049 & -------- & & 1 & & & & \\
\hline Sterile & -------- & ------- & & & 1 & & & \\
\hline Sterile & ITCS050 & -------- & 1 & & & & & \\
\hline Productive & ITCS051 & -------- & & 13 & & & & \\
\hline Sterile & ITCS051 & -------- & 9 & & & & & \\
\hline Sterile & -------- & -------- & & & 1 & & & \\
\hline Sterile & ITCS050 & -------- & 8 & & & & & \\
\hline Productive & ITCS050 & -------- & & 7 & & & & \\
\hline Sterile & -------- & -------- & & & 1 & & & \\
\hline Sterile & ITCS012 & -------- & 1 & & & & & \\
\hline Productive & -------- & -------- & & & & 13 & & \\
\hline Sterile & -------- & -------- & & & 7 & & & \\
\hline Sterile & -------- & ------- & & & 1 & & & \\
\hline
\end{tabular}




\section{Well name}

Spinazzino 1

Spinazzino 2, 3, 4

Torri 1

Trava 1

Travasona 1

Travasona 2

Tre Motte 2

Tre Motte 3

Tresigallo 2, 5, 14, 15

Tresigallo 3, 4, 6, 7, 8, 9-13

Valle Isola 1

Valle Isola 1

Valle Lepri 1

Vallesina 1

Valletta 1

Valli di Comacchio 1, 2, 3

Valli di Comacchio Nord Ovest 1, 2

Valli di Comacchio NW 1, 2

Vallicella 1

Varano 1

Vicenza Nuova 1

Volano 1

Vulcano

Zorabini 1,2

\begin{tabular}{|c|c|}
\hline $\begin{array}{c}\text { Well } \\
\text { category }\end{array}$ & $\begin{array}{l}\text { Falls inside } \\
\text { CSS }\end{array}$ \\
\hline Ambiguous $^{\circ 0}$ & ITCS012 \\
\hline Sterile & ITCS012 \\
\hline Sterile & -------- \\
\hline Sterile & ITCS012 \\
\hline Ambiguous $^{\circ}$ & -------- \\
\hline Sterile & ------- \\
\hline Ambiguous $^{\circ}$ & -------- \\
\hline Productive & ITCS012 \\
\hline Productive & ITCS050 \\
\hline Sterile & ITCS050 \\
\hline Sterile & ------- \\
\hline Sterile & ----- \\
\hline Sterile & ----- \\
\hline Sterile & ------- \\
\hline Sterile & --------- \\
\hline Sterile & ITCS012 \\
\hline Sterile & ITCS012 \\
\hline Sterile & --------- \\
\hline Ambiguous $^{\circ}$ & ITCS050 \\
\hline Sterile & -------- \\
\hline Sterile & ITCS050 \\
\hline Sterile & -------- \\
\hline Sterile & ITCS012 \\
\hline Ambiguous $^{\circ}$ & ITCS012 \\
\hline
\end{tabular}
insid

Sterile CSS

css

Sterile

Productive

Sterile Productive

--------

3

1

1

10

1

1

1

2

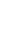

1

1

145

116

63

74
ISS

exploration borehole which revealed a gas/oil reservoir (never into production)

$\circ$ gas reservoir shallower than $500 \mathrm{~m}$

[ multiple wells 